\title{
Two methods to forecast auroral displays
}

\author{
Fred Sigernes ${ }^{1, *}$, Margit Dyrland ${ }^{1}$, Pål Brekke ${ }^{2}$, Sergey Chernouss ${ }^{3}$, Dag Arne Lorentzen ${ }^{1}$, Kjellmar Oksavik $^{1}$, and \\ Charles Sterling Deehr ${ }^{4}$ \\ 1 The University Centre in Svalbard (UNIS), N-9171 Longyearbyen, Norway \\ *corresponding author: e-mail: freds@unis.no \\ 2 Norwegian Space Centre, Oslo, Norway \\ 3 Polar Geophysical Institute, Murmansk Region, Apatity, Russia \\ 4 Geophysical Institute, University of Alaska, Fairbanks, USA
}

Received 5 May 2011 / Accepted 6 September 2011

\begin{abstract}
This work compares the methods by Starkov (1994a) and Zhang \& Paxton (2008), that calculate the size and location of the auroral ovals as a function of planetary $K p$ index. The ovals are mapped in position and time onto a solar illuminated surface model of the Earth. It displays both the night- and dayside together with the location of the twilight zone as Earth rotates under the ovals. The graphical display serves as a tool to forecast auroral activity based on the predicted value of the $K p$ index. The forecast is installed as a service at http://kho.unis.no/. The Zhang \& Paxton (2008) ovals are wider in latitude than the Starkov (1994a) ovals. The nightside model ovals coincide fairly well in shape for low to normal auroral conditions. The equatorward border of the diffuse aurora is well defined by both methods on the nightside for $K p \leq 7$. The dayside needs further studies in order to conclude.
\end{abstract}

Key words. 2704: auroral phenomena - 1922: forecasting - 2753: numerical modeling

\section{Introduction}

On Earth the impact zones of energetic particles from the Sun, i.e. a circular belt of auroral emissions around each geomagnetic pole, are known as the auroral ovals (cf. Akasofu 1964). The location and size of these ovals have been studied extensively in the second half of the 20th century.

During the International Geophysical Year (1957-1958), auroral occurrence was determined from all-sky camera studies for a wide variety of activity levels. The resulting figure was made up from a statistical study showing the poleward and equatorward auroral boundaries of the $75 \%$ occurrence probability (Feldstein 1963, 1973; Feldstein \& Starkov 1970). The relationship between the morphology of the auroral oval and the level of geomagnetic activity allows us to develop models of the location of the aurora, independent of the vagaries of auroral observations (Holzworth \& Meng 1975; Starkov 1994a).

Over the last three decades, new models have evolved that use data of particle precipitation measured by polar orbiting satellites (Gussenhoven et al. 1983; Hardy \& Gussenhoven 1985; Hardy et al. 1987, 1989). Recently, Zhang \& Paxton (2008) developed an auroral oval model based on data from the Ultraviolet Imager (GUVI) on board the TIMED (Thermosphere Ionosphere Mesosphere Energetics and Dynamics) satellite.

The planetary geomagnetic activity index $K p$ (Bartels et al. 1939 ) is directly related to the size and location of the auroral oval, and it is used as an input parameter to the above models. The $K p$ index is provided by the Helmholtz Centre in Potsdam, and it involves worldwide data collection and careful interpretation in line with a tradition that began in 1936 . The traditional $K p$ index is not reported in real time. It is therefore not useful for forecasting purposes. However, an intermediate method of scaling the $K p$ from a small number of midlatitude stations has been developed by the US Air Force 55th Space Weather Squadron. This alternative $K p$ index is referred to as the estimated $K p$ index (Takahashi et al. 2001), and it will be used in the present work.

The advent of stationary spacecraft between the Sun and the Earth has led to studies of the relationship between the structure of the solar wind and the resulting auroral and geomagnetic disturbances. When the input data come from a satellite located approximately $1 \mathrm{~h}$ upstream in the solar wind, the resultant predicted $K p$ forecast is relatively short term (Costello 1997; Wing et al. 2005). It gives a 1- or 4-h warning. This can be very useful both for auroral observers and experiments that are dependent on the location and intensity of the aurora.

In this study we will compare the models of Starkov (1994a) and Zhang \& Paxton (2008). Our goal is to produce a real-time forecast, up to $1 \mathrm{~h}$ in advance, for the location and size of the aurora oval and mapped onto the Earth's surface. Solar elevation and cloud cover are the main obstacles for an auroral observer at the ground level. Therefore, this forecast also includes the observing conditions for a particular site. To our knowledge, no such combined display of information currently exists.

\section{Two models of the auroral oval}

This section describes the auroral oval models of Starkov (1994a) and Zhang \& Paxton (2008). Both models are presented using the same mathematical notation.

\subsection{The Feldstein-Starkov ovals}

Starkov (1994a) compiled simple formulas of the poleward, equatorward and diffuse auroral boundaries as a function of magnetic activity.

The magnetic input parameter is the $A L$ index. This index describes the polar or planetary magnetic disturbances that 
Table 1. Coefficients to convert $K p$ to $A L$ indices by Starkov (1994b) used by equation (1) in units of nT.

\begin{tabular}{cccc}
\hline \hline$c_{0}$ & $c_{1}$ & $c_{2}$ & $c_{3}$ \\
\hline 18 & -12.3 & 27.2 & -2.0 \\
\hline
\end{tabular}

occur during auroras. The range of the index is of the order of \pm $800 \mathrm{nT}$. In terms of $K p$ index it is given as (Starkov 1994b)

$$
A L=c_{0}+c_{1} \cdot K_{p}+c_{2} \cdot K_{p}^{2}+c_{3} \cdot K_{p}^{3}
$$

The coefficients $c_{i}$ for $i \in[0, \ldots, 3]$ are given in Table 1 .

The value of the $K p$ index varies from 0 to 9 with 0 being very quiet and 5 or more indicating geomagnetic storm conditions. In detail, $K p$ represents a 3 -h weighted average from a global network of magnetometers measuring the maximum deviation in the horizontal component of the Earth's magnetic field.

In coordinates of corrected geomagnetic colatitude, $\theta$, the boundaries of the oval are expressed as

$$
\begin{aligned}
\theta_{m}= & A_{0 m}+A_{1 m} \cos \left[15\left(t+\alpha_{1 m}\right)\right] \\
& +A_{2 m} \cos \left[15\left(2 t+\alpha_{2 m}\right)\right]+A_{3 m} \cos \left[15\left(3 t+\alpha_{3 m}\right)\right]
\end{aligned}
$$

where $A_{i m}$ and $\alpha_{i m}$ for $i \in[0, \ldots, 3]$ are amplitudes in units of degrees of latitude and phases in units of decimal hours, respectively. $t$ is local time. Equation (2) is valid for the poleward $(m=0)$, the equatorward $(m=1)$ and the diffuse aurora boundaries $(m=2)$. The challenge now is to find the coefficients of equation (2).

Starkov (1994a) does this using a new third-order polynomial for both the $A_{i m}$ and $\alpha_{i m}$ coefficients

$$
\begin{gathered}
A_{\text {im }} \text { or } \alpha_{i m}=b_{0 m}+b_{1 m} \log _{10}|A L|+ \\
\quad+b_{2 m} \log _{10}^{2}|A L|+b_{3 m} \log _{10}^{3}|A L|
\end{gathered}
$$

In other words, for each coefficient $A_{i m}$ or $\alpha_{i m}$ there is a set of $b_{i m}$ values, $i \in[0, \ldots, 3]$, for each boundary with index $m$. Appendix A lists all the values.

Starting with the $K p$ index, equation (1) is used to obtain the $A L$ index. Next, Appendix $\mathrm{A}$ is used to obtain the correct set of $b_{i m}$ values to calculate the $A_{i m}$ or $\alpha_{i m}$ coefficients by equation (3). Finally, the auroral boundaries are obtained using equation (2). Note that the procedure works for $K p$ indices that are floating point numbers. The ovals are expanding smoothly as the $K p$ index increases.

\subsection{The Zhang-Paxton ovals}

This model uses Epstein functions to calculate either the electron flux or the mean energy flux for precipitating electrons based on data from the GUVI on board the TIMED satellite. The electron energy flux $\left[\operatorname{ergs~} \mathrm{cm}^{-2} \cdot \mathrm{s}^{-1}\right.$ ] is given as

$$
Q_{m}=\frac{A_{0 m}^{\prime} \cdot \exp \left[\left(x-A_{1 m}^{\prime}\right) / A_{2 m}^{\prime}\right]}{\left\{1+\exp \left[\left(x-A_{1 m}^{\prime}\right) / A_{3 m}^{\prime}\right]\right\}^{2}}
$$

where $x=\pi / 2-|\theta|$ is the comagnetic latitude. The index $m \in$ $[0, \ldots, 5]$ refers to six subintervals of $K p$ index with center values $k_{m}=[0.75,2.25,3.75,5.25,7.00,9.00]$. The coeffi-

\begin{tabular}{|c|c|c|c|c|c|}
\hline$\left[\mathrm{ergs} \mathrm{cm}^{-2} \cdot \mathrm{s}^{-1}\right.$ ] & $\bar{c} c_{0}^{\prime}$ & $\overline{c_{1}^{\prime}}$ & $c_{2}^{\prime}$ & $c_{3}^{\prime}$ & $c_{4}^{\prime}$ \\
\hline$K_{p} \leq 7$ & 1.40 & -0.19 & 0.83 & -0.21 & 0.018 \\
\hline $7 \leq K_{p} \leq 9$ & 24.74 & 8.73 & -3.08 & 0.225 & 0.000 \\
\hline
\end{tabular}
cients $A_{i m}^{\prime}$ for $i \in[0, \ldots, 3]$ are calculated by a Fourier series

$$
\begin{aligned}
& A_{i m}^{\prime}=b_{0 m}^{\prime}+\sum_{n=1}^{6}\left\{b_{n m}^{\prime} \cos \left(\frac{n \pi t}{12}\right)+b_{n m}^{\prime \prime} \sin \left(\frac{n \pi t}{12}\right)\right\} \\
& b_{n m}^{\prime} \text { and } b_{n m}^{\prime \prime} \text { for } n \in[0, \ldots, 6] \text { are listed in Appendix B. }
\end{aligned}
$$

Table 2. Coefficients to find maximum electron energy flux as a function of $K p$ index from the Zhang-Paxton model, used in equation (11).

The electron flux, $Q$, at any $K p$ index, can then be found using a nonlinear interpolation

$$
Q=f_{m} \times Q_{m}+f_{m+1} \times Q_{m+1}
$$

where the subscripts $m$ and $m+1$ refer to the closest subintervals that satisfy the conditions

$$
k_{m} \leq K p \leq k_{m+1}
$$

Note that if $K p$ is less than 0.75 then $k_{0}=0.75$ and $k_{1}=2.25$. Furthermore, the scale factors $f_{m}$ and $f_{m+1}$ in equation (6) are calculated as

$$
f_{m}=\frac{H P\left(k_{m+1}\right)-H P(K p)}{H P\left(k_{m+1}\right)-H P\left(k_{m}\right)}
$$

and

$$
f_{m+1}=\frac{H P(K p)-H P\left(k_{m+1}\right)}{H P\left(k_{m+1}\right)-H P\left(k_{m}\right)}
$$

The function $H P$ is the auroral hemispheric power as a function of $K p$ index in units of gigawatts

$$
H P(K p)=\left\{\begin{array}{l}
38.66 \exp (0.1967 K p) K p \leq 5.0 \\
4.592 \exp (0.4731 K p) K p>5.0
\end{array}\right.
$$

In order to use the model it is necessary to scale the output. For each model run, the maximum value of the electron energy flux is stored. The net result is a polynomial fit that gives the maximum flux as a function of $K p$ index

$$
Q_{\max }=c_{0}^{\prime}+c_{1}^{\prime} K_{p}+c_{2}^{\prime} K_{p}^{2}+c_{3}^{\prime} K_{p}^{3}+c_{4}^{\prime} K_{p}^{4}
$$

The coefficients $c_{i}^{\prime}$ for $i \in[0 \ldots 4]$ are given in Table 2 .

\section{Geographical transform}

The ovals calculated in the previous section are centered on the magnetic poles (dipole). The Cartesian components in the graph are related to the polar magnetic coordinates by

$$
\begin{aligned}
& x_{m}=\sin \theta \cdot \cos \phi \\
& y_{n}=\sin \theta \cdot \cos \phi \\
& z_{m}=\cos \theta
\end{aligned}
$$

where $\theta$ and $\phi$ are the magnetic latitude and longitude, respectively. In local magnetic time it becomes

$$
\phi=2 \pi \cdot t / 24+\Delta \phi(t)
$$

where $\Delta \phi(t)$ is the longitudinal difference between the subsolar point and the magnetic poles at time $t$ (hours). It is important in order to make sure that the ovals are oriented correctly with magnetic noon pointing toward the Sun as Earth rotates around its own axis.

The transformation to geographic coordinates is then

$$
\left[\begin{array}{c}
x \\
y \\
z
\end{array}\right]=\tilde{\mathbf{R}} \cdot\left[\begin{array}{l}
x_{m} \\
y_{m} \\
z_{m}
\end{array}\right]
$$


where $\tilde{\mathbf{R}}$ is a rotational matrix. The invariant magnetic north pole is located in geographic coordinates at latitude $\theta_{0}^{\prime}=82.41^{\circ} \mathrm{N}$ and longitude $\phi_{0}^{\prime}=-82.86^{\circ} \mathrm{E}$.

The elements of the rotational matrix are then simply

$$
\tilde{\mathbf{R}}=\left[\begin{array}{ccc}
\cos \phi_{0}^{\prime} \cos \lambda & -\sin \phi_{0}^{\prime} & \cos \phi_{0}^{\prime} \sin \lambda \\
\sin \phi_{0}^{\prime} \cos \lambda & \cos \phi_{0}^{\prime} & \sin \phi_{0}^{\prime} \sin \lambda \\
-\sin \lambda & 0 & \cos \lambda
\end{array}\right]
$$

where $\lambda=\pi / 2-\theta_{0}^{\prime}$ is the latitudinal difference between the geographic and the north magnetic pole.

Finally, the geographic latitude and longitude of the ovals are given as

$$
\begin{gathered}
\theta^{\prime}=\frac{\pi}{2}-\cos ^{-1}(z) \\
\psi=\tan ^{-1}(y / x) \\
\phi^{\prime}= \begin{cases}\psi \forall & x>0 \\
\psi+\pi & x<0\end{cases}
\end{gathered}
$$

Note that the procedure is identical for the south magnetic pole if we assume that the ovals are mirrored.

\section{Visualization}

The ovals are visualized with a stand-alone 32-bit executable Windows program called SvalTrack II. The program is written in Borland's Delphi-Pascal and uses a Geographic Information System unit that displays interactively mapped data in real time onto a three-dimensional spherical Globe representing the Earth. The twilight zone, night- and dayside of the Earth are projected with grades of shade on the Globe as a function of time. The 3D globe can be rotated and zoomed to display a close-up of any region of the Earth.

The Feldstein-Starkov ovals are projected as semitransparent polygons onto the globe with an angular resolution of $1.5^{\circ}$. The equatorward boundary of the diffuse aurora is added as a polygonal line. The Zhang-Paxton ovals are mapped onto the globe with transparent scaled intensities according to electron energy flux. Every pixel of the display is processed in real time to determine electron energy flux values or which colors to draw onto the surface of the globe.

The local observational position is added as a point (red triangle) with corresponding state information of the Moon and the Sun. In addition, the circle of $\sim 10^{\circ}$ around the observer represents a $180^{\circ}$ field of sky view. The latter is under the assumption that the auroral emissions peak at an altitude of $\sim 110 \mathrm{~km}$.

All the above features are shown in Figure 1. A textured map is used to visualize the Earth on 24th December 2009. The twilight zone crosses most of Norway, and North America is on the nightside. The observer is located in Longyearbyen $\left(78.2^{\circ} \mathrm{N}, 16.0^{\circ} \mathrm{E}\right)$, on the archipelago Svalbard, Norway. The $K p=3$ oval is typically broader on the night- vs. the dayside, with magnetic noon or cusp located over the site at $\sim 08: 50$ UT. The Moon is below the horizon, indicating favorable conditions to view the aurora. Also note that the island of Svalbard is uniquely located. It is possible to view both the day- and nightside aurora from this location midwinter.
(A)
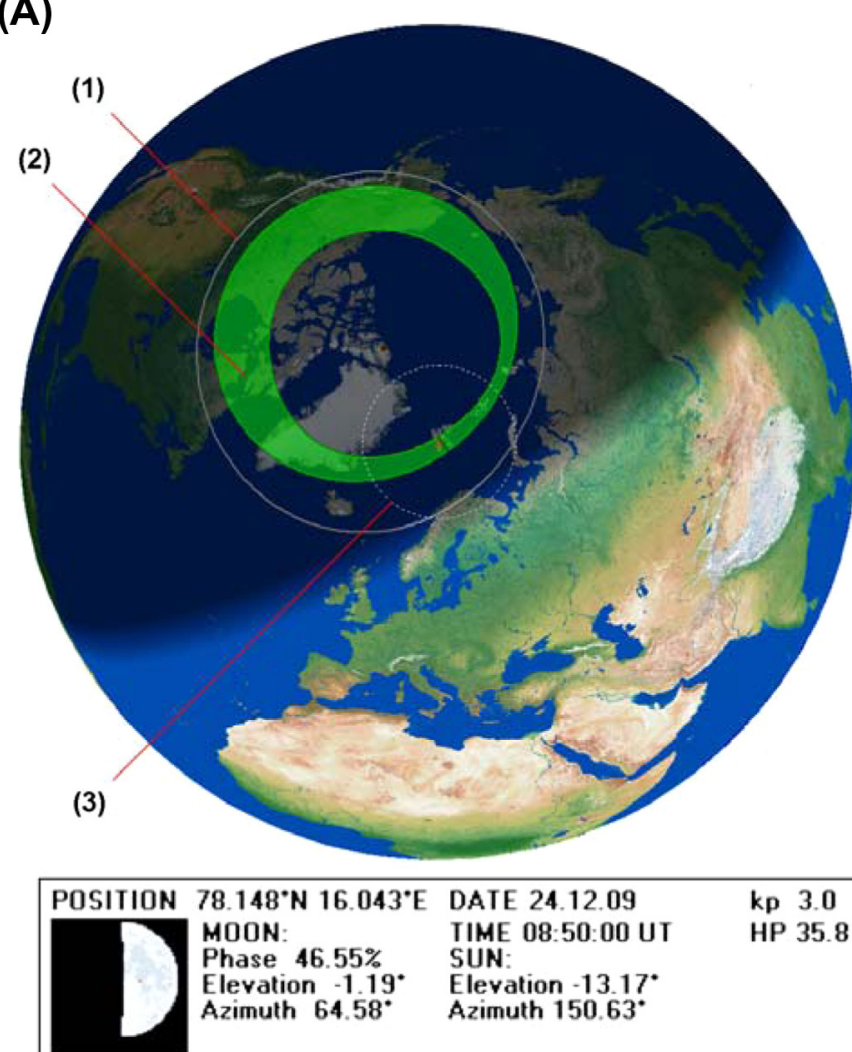

OPTICS +

Fig. 1. SvalTrackII screen dumps: Panels (A) and (B) show the ovals by Starkov (1994a) and Zhang \& Paxton (2008), respectively. (1) Equatorward boundary of the diffuse aurora, (2) Feldstein-Starkov aurora oval, (3) field of view aurora observer, (4) is Zhang-Paxton oval, (5) observer location, (6) Moon and Sun information at local site. Time: 08:50 UT on 24th December 2009 . The Kp index equals 3.
(B)

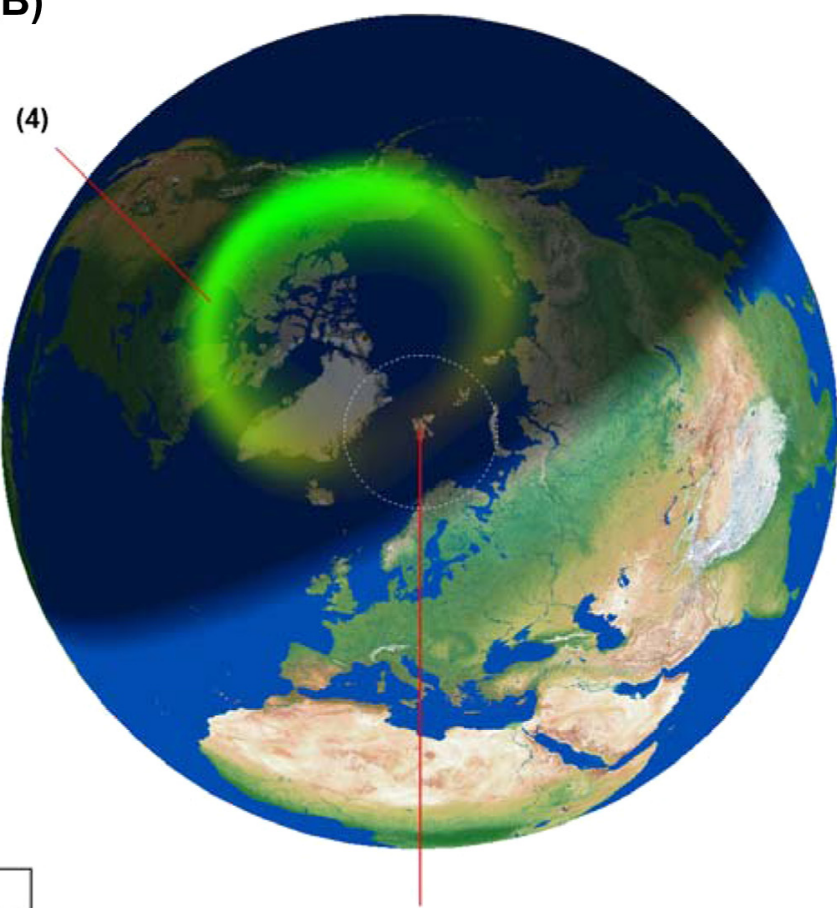

(5) 

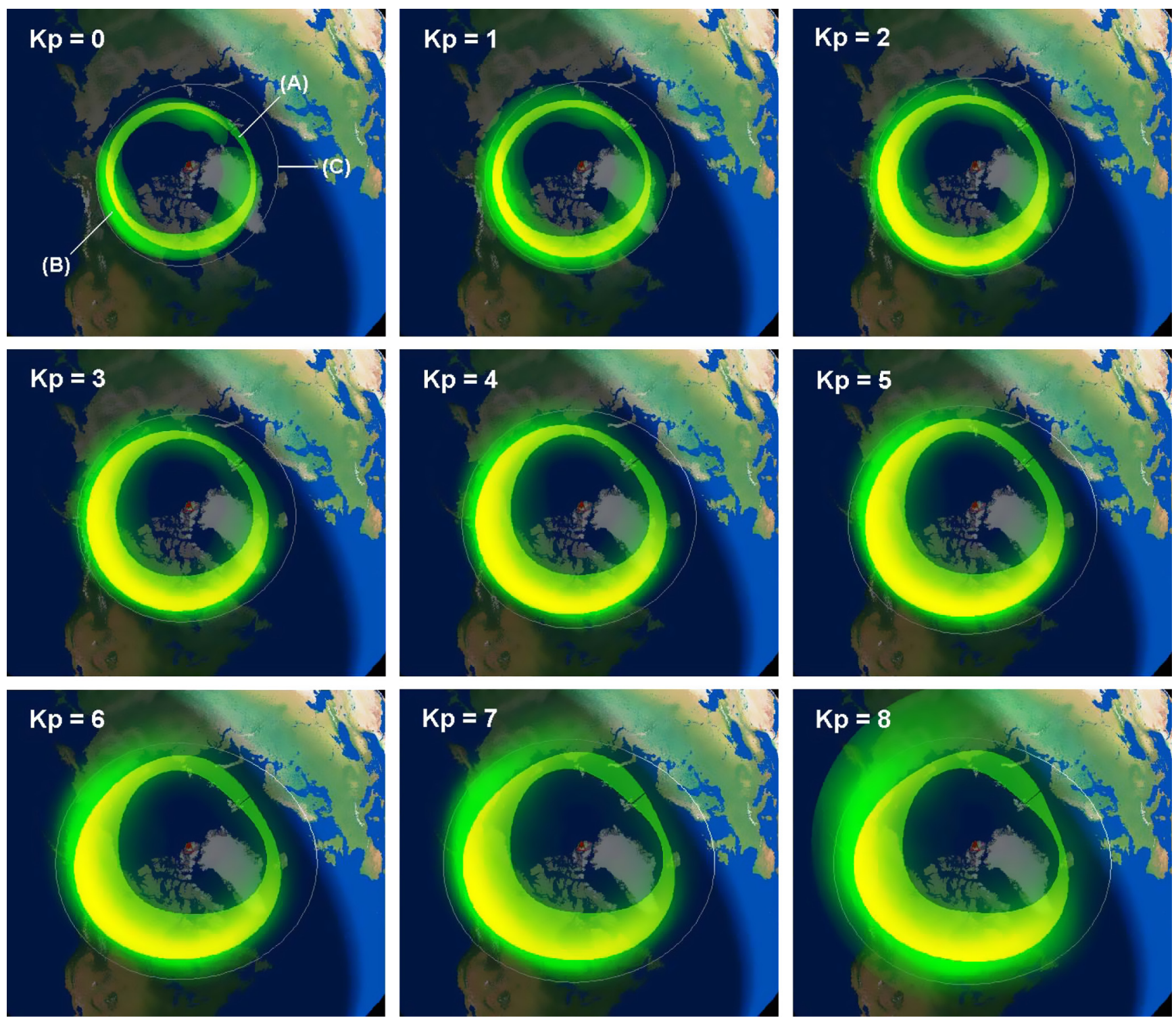

Fig. 2. Animated model aurora ovals as a function of $K p$ index for 24th December 2009 at 08:50 UT. The transparent polygons represent Feldstein-Starkov ovals (A). The faint white outer ring is the equatorward boundary of the diffuse aurora (C). The Zhang-Paxton ovals are displayed on top with green intensity values scaled according to the electron energy flux (B). The yellow scaled intensity areas are the intersection $(\mathrm{A} \cap \mathrm{B})$ between the two models.

Table 3. Auroral intersection (A $\cap \mathrm{B}$ ) between the Zhang-Paxton (A) and the Feldstein-Starkov (B) ovals as a function of $K p$ index. (A $\cap \mathrm{C})$ is the interception between (A) and the equatorward boundary of diffuse aurora from Starkov (1994a) (C). $Q_{\max }$ is the maximum electron flux. $Q_{\text {min }}=0.25 \mathrm{ergs} \mathrm{cm}^{-2} \cdot \mathrm{s}^{-1}$.

\begin{tabular}{llcccc}
\hline \hline$K p$ & Auroral activity & Level & $\mathrm{A} \cap \mathrm{B}[\%]$ & $\mathrm{A} \cap \mathrm{C}[\%]$ & $Q_{\mathrm{max}}\left[\mathrm{mW} \cdot \mathrm{m}^{-2}\right]$ \\
\hline 0 & Minimum & Normal conditions & 32 & 99 & 1.65 \\
1 & Quiet & & 26 & 86 & 2.10 \\
2 & Low & & 33 & 87 & 3.20 \\
3 & Moderate & 35 & 89 & 4.34 \\
4 & Active & High activity levels & 36 & 88 & 5.34 \\
5 & High & & 35 & 84 & 8.45 \\
6 & High + & & 32 & 83 & 12.18 \\
7 & High ++ & & 24 & 68 & 12.91 \\
8 & High +++ & 22 & 62 & 18.10 \\
9 & Maximum & & & & \\
\hline
\end{tabular}

The only inputs to the above visualizations are time and the $K p$ index. The program is set to update itself in real time based on the predicted $K p$ index provided by the Space Weather
Prediction Centre at the National Oceanic and Atmospheric Administration (NOAA). 
The index is predicted every $15 \mathrm{~min}$ by the use of the Wing $K p$ Predicted Activity Index model (Wing et al. 2005), which takes into account the most recent estimated $K p$ values and the response of solar wind parameters (neural network algorithm).

The result is a real-time auroral oval forecast up to approximately 1 or $4 \mathrm{~h}$ in advance. The software is installed to run automatically at the Kjell Henriksen Observatory (KHO) on Svalbard, Norway. See link: http://kho.unis.no/.

\section{Model comparision}

Figure 2 shows the results from the two models plotted on top of each other for different $K p$ indices. As expected, both models show ovals that are wider and more asymmetric with increasing $K p$ index. The Zhang-Paxton ovals have a larger latitudinal spread than the Feldstein-Starkov ovals, but they are close and within the equatorward boundary of the diffuse aurora of Starkov (1994a) on the nightside, except for severe and extreme auroral storm conditions. The spread in latitude is seen as a diffuse effect on the ovals of Figure 2. The main difference between the model oval shapes is for storm conditions, where the Feldstein-Starkov dusk side of the ovals is poleward of the Zhang-Paxton ovals.

In order to quantify the difference, we define the intersection between the modeled ovals as the percentage fraction of the Zhang-Paxton points that are inside the Feldstein-Starkov ovals (polygons). The yellow glow of Figure 2 represents the intersection. Table 3 lists the results. Note that the overall intersection percentage is quite low ( $\mathrm{A} \cap \mathrm{B}<36 \%$ ). This is due to the diffuse effect mentioned earlier. The Zhang-Paxton ovals are based on data from spaceborne ultraviolet cameras. These cameras see radiation that is not detected by ground-based instruments. This is due to the ultraviolet absorption in the middle atmosphere. Diffuse aurora equatorward of the discrete/visible aurora, caused by high energy particles that are lost from the ring current/radiation belts, may be more visible from space than from the ground.

On the other hand, the intersection $\mathrm{A} \cap \mathrm{C}>80 \%$ for $K p \leq$ 7. The diffuse equatorward border of Starkov (1994a) is close to the Zhang \& Paxton (2008) equatorward oval border on the nightside. On the dayside the situation is opposite. The Starkov (1994a) diffuse border is clearly equatorward of the Zhang \& Paxton (2008) equatorward oval border. We suspect that the lack or quality of the data from the dayside region both from space or ground may be the reason for the difference. On the dayside, the oval is contaminated by ultraviolet radiation from the Sun, and from the ground there are fewer observations compared to the nightside.

Note that this study uses the same threshold electron flux as Zhang \& Paxton (2008), $Q_{\text {min }}=0.25 \mathrm{ergs}^{-2} \cdot \mathrm{sm}^{-1}$. Raising the threshold value will cut low flux contributions both poleward and equatorward of the ovals. The intersection of the ovals will then increase correspondingly. If we set the threshold too high, then the intersection also becomes too high. An intersection that equals $100 \%$ may only plot high flux values inside a small portion of the Feldstein-Starkov ovals. The relation between the threshold as a function of $K p$ index and what is detectable from ground is an aim for future studies.

\section{Concluding remarks}

The two methods by Starkov (1994a) and Zhang \& Paxton (2008) are used to mathematically calculate the size and location of the auroral oval mapped onto a solar illuminated Earth globe. Both models only use $K p$ index and time as input, which makes them ideal candidates for forecasting aurora when the predicted $K p$ value is estimated from satellite data 1 hour upstream in the solar wind. The forecast is found at http://kho.unis.no/. As expected the Zhang-Paxton ovals deduced by space-borne data are wider than the ground-based Feldstein-Starkov ovals. In spite of difference in methods and platforms, the model ovals coincide fairly well in shape for low to normal conditions on the nightside. In addition, the equatorward border of the diffuse aurora is well defined by both methods on the nightside for $K p \leq 7$. On the dayside, there is a need to study further oval shapes for all levels of auroral activity, especially the equatorward border of the diffuse aurora.

Acknowledgements. We wish to thank the National Oceanic and Atmospheric Administration - Space Weather Prediction Centre for allowing us to download the predicted value of the estimated $K p$ index every $15 \mathrm{~min}$. The work is financially supported by The Research Council of Norway through the project named: Norwegian and Russian Upper Atmosphere Co-operation On Svalbard part 2 \# 196173/S30 (NORUSCA2), the Nordic Council of Ministers: Arctic Cooperation Programme \# A10162, and COST action ES0803.

\section{References}

Akasofu, S.-I., The latitudinal shift of the auroral belt, J. Atm. Terr. Phys., 26, 1167-1174, 1964.

Bartels, J., N.H. Heck, and H.F. Johnston, The three-hour range index measuring geomagnetic activity, J. Geophys. Res., 44, 411454, 1939.

Costello, K.A., Moving the Rice MSFM into a Real-Time Forecast Mode Using Solar Wind Driven Forecast Models, Ph.D. dissertation, Rice University, Houston, TX, June 1997.

Feldstein, Y.I., On morphology of auroral and magnetic disturbances at high latitudes, Geomag. Aeron., 3, 183-192, 1963.

Feldstein, Y.I., Auroral oval, J. Geophys. Res., 78 (7), 1210-1213, 1973.

Feldstein, Y.I., and G.V. Starkov, The auroral oval and the boundary of closed field lines of the geomagnetic field, Planet. Space Sci., 18, 501-508, 1970.

Gussenhoven, M.S., D.A. Hardy, and N. Heinemann, Systematics of the equatorward diffuse auroral boundary, J. Geophys. Res., 88, 5692-5708, 1983.

Hardy, D.A., and M.S. Gussenhoven, A statistical model of auroral electron precipitation, J. Geophys. Res., 90 (A5), 4229-4248, 1985.

Hardy, D.A., M.S. Gussenhoven, and R. Raistrick, Statistical and functional representations of pattern of auroral energy flux, number flux, and conductivity, J. Geophys. Res., 92 (A11), 12275-12294, 1987.

Hardy, D.A., M.S. Gussenhoven, and D. Brautigam, A statistical model of auroral ion precipitation, J. Geophys. Res., 94 (A1), 370-392, 1989.

Holzworth, R.H., and C.I. Meng, Mathematical representation of the auroral oval, Geophys. Res. Lett., 2, 377-380, 1975.

Starkov, G.V., Mathematical model of the auroral boundaries, Geomag. Aeron., 34 (3), 331-336, 1994a.

Starkov, G.V., Statistical dependences between the magnetic activity indices, Geomag. Aeron., 34 (1), 101-103, 1994b.

Takahashi, K., B. Toth, and J. Olson, An automated procedure for near-real-time $K p$ estimates, J. Geophys. Res., 106 (A10), 2101721032, 2001.

Wing, S., J.R. Johnson, J. Jen, C.-I. Meng, D.G. Sibeck, K. Bechtold, J. Freeman, K. Costello, M. Balikhin, and K. Takahashi, Kp forecast models, J. Geophys. Res., 110, A04203, DOI: 10.1029/ 2004JA010500, 2005.

Zhang, Y., and L.J. Paxton, An empirical Kp-dependent global auroral model based on TIMED/GUVI data, J. Atm. Solar-Terr. Phys., 70, 1231-1242, 2008. 


\section{Appendix A}

Expansion coefficients for auroral boundaries by Starkov (1994a) used in equation (3).

\begin{tabular}{|c|c|c|c|c|c|c|c|}
\hline Units & {$\left[{ }^{\circ}\right]$} & {$\left[{ }^{\circ}\right]$} & {$\left[{ }^{0}\right]$} & {$\left[{ }^{0}\right]$} & {$[\mathrm{h}]$} & {$[\mathrm{h}]$} & {$[\mathrm{h}]$} \\
\hline$m=0$ & \multicolumn{6}{|c|}{$m=0$ Poleward boundary of the auroral oval } & $\alpha_{30}$ \\
\hline$b_{00}$ & -0.07 & -10.06 & -4.44 & -3.77 & -6.61 & 6.37 & -4.48 \\
\hline$b_{10}$ & 24.54 & 19.83 & 7.47 & 7.90 & 10.17 & -1.10 & 10.16 \\
\hline$b_{20}$ & -12.53 & -9.33 & -3.01 & -4.73 & -5.80 & 0.34 & -5.87 \\
\hline$b_{30}$ & 2.15 & 1.24 & 0.25 & 0.91 & 1.19 & -0.38 & 0.98 \\
\hline & \multicolumn{6}{|c|}{$m=1$ Equatorward boundary of the auroral oval } & $\alpha_{31}$ \\
\hline$b_{01}$ & 1.61 & -9.59 & -12.07 & -6.56 & -2.22 & -23.98 & -20.07 \\
\hline$b_{11}$ & 23.21 & 17.78 & 17.49 & 11.44 & 1.50 & 42.79 & 36.67 \\
\hline$b_{21}$ & -10.97 & -7.20 & -7.96 & -6.73 & -0.58 & -26.96 & -24.20 \\
\hline \multirow[t]{2}{*}{$b_{31}$} & 2.03 & 0.96 & 1.15 & 1.31 & 0.08 & 5.56 & 5.11 \\
\hline & $A_{02}$ & $A_{12}$ & $A_{22}$ & $A_{32}$ & $\alpha_{12}$ & $\alpha_{22}$ & $\alpha_{32}$ \\
\hline \multicolumn{8}{|c|}{$m=2$ Equatorward boundary of the diffuse aurora } \\
\hline$b_{02}$ & 3.44 & -2.41 & -0.74 & -2.12 & -1.68 & 8.69 & 8.61 \\
\hline$b_{12}$ & 29.77 & 7.89 & 3.94 & 3.24 & -2.48 & -20.73 & -5.34 \\
\hline$b_{22}$ & -16.38 & -4.32 & -3.09 & -1.67 & 1.58 & 13.03 & -1.36 \\
\hline$b_{32}$ & 3.35 & 0.87 & 0.72 & 0.31 & -0.28 & -2.14 & 0.76 \\
\hline
\end{tabular}

\section{Appendix B}

Epstein-Fourier coefficients by Zhang \& Paxton (2008) used in equation (5).

\begin{tabular}{|c|c|c|c|c|}
\hline Units & {$\left[\mathrm{ergs} \mathrm{cm}^{2} \cdot \mathrm{s}^{-1}\right]$} & {$\left[^{\circ}\right]$} & {$\left[{ }^{\circ}\right]$} & $\left.{ }^{\circ}\right]$ \\
\hline$m=0$ & $=0.75^{A_{00}^{\prime}}$ & $A_{10}^{\prime}$ & $A_{20}^{\prime}$ & $A_{30}^{\prime}$ \\
\hline$b_{00}^{\prime}$ & 3.2402978 & 18.221647 & 2.8442195 & 1.8336810 \\
\hline$b_{10}^{\prime}$ & 1.3291109 & 6.0779378 & 0.2931214 & -0.3873319 \\
\hline$b_{20}^{\prime 10}$ & 0.4209809 & -1.3235271 & -0.5383766 & 0.0094550 \\
\hline$b_{30}^{\prime}$ & 0.4779546 & 0.2553541 & 0.2728634 & 0.0548413 \\
\hline$b_{40}^{\prime}$ & -0.3673774 & 0.0980007 & 0.2950094 & -0.0113930 \\
\hline$b_{50}^{\prime 40}$ & 0.0557991 & 0.0075817 & -0.0456968 & -0.0434681 \\
\hline$b_{60}^{\prime}$ & -0.0556684 & -0.0050137 & 0.0073026 & 0.0562728 \\
\hline$b_{10}^{\prime \prime}$ & -0.1914775 & 1.3812608 & 0.8133630 & 0.1214365 \\
\hline$b_{20}^{1 \prime \prime}$ & 0.0802700 & -0.0211661 & -0.3734801 & -0.2901524 \\
\hline$b_{30}^{20}$ & -0.4206442 & -0.1240047 & -0.0874247 & 0.2791531 \\
\hline$b_{40}^{3 \prime \prime}$ & -0.0354484 & 0.3415960 & -0.0607254 & -0.1764180 \\
\hline $\begin{array}{l}40 \\
b_{50}^{\prime \prime}\end{array}$ & -0.2017480 & -0.1074103 & 0.1679693 & 0.0400522 \\
\hline$b_{60}^{\prime \prime \prime}$ & -0.0298214 & 0.2863931 & -0.0456554 & -0.1214534 \\
\hline & $A_{01}^{\prime}$ & $A_{11}^{\prime}$ & $A_{21}^{\prime}$ & $A_{31}^{\prime}$ \\
\hline \multicolumn{5}{|c|}{$m=1(K p: 1.5-3.0) k_{1}=2.25^{101}$} \\
\hline$b_{01}^{\prime}$ & 6.3011691 & 19.882410 & 2.9663179 & 1.8560835 \\
\hline$b_{11}^{\prime}$ & 3.2522103 & 6.5577617 & 0.8165399 & -0.3102606 \\
\hline$b_{21}^{\prime 1}$ & -0.2114976 & -1.5086115 & -0.3559603 & 0.0363061 \\
\hline$b_{31}^{\prime 1}$ & 0.3216365 & 0.5457940 & 0.3101074 & 0.0574751 \\
\hline$b_{41}^{\prime}$ & -0.3236900 & -0.0926016 & 0.0969373 & -0.0304515 \\
\hline$b_{51}^{\prime 1}$ & 0.1705425 & -0.0363445 & -0.0530104 & 0.0657831 \\
\hline$b_{61}^{\prime 1}$ & 0.0919870 & -0.0801630 & -0.0685254 & 0.0046886 \\
\hline$b_{11}^{\prime \prime}$ & -0.6934043 & 1.6156140 & 0.7957589 & -0.0660346 \\
\hline$b_{21}^{\prime \prime \prime}$ & -0.1779372 & -0.0385058 & -0.4427029 & -0.3614867 \\
\hline$b_{31}^{211}$ & -0.3675894 & -0.1844160 & -0.1514768 & 0.1820734 \\
\hline$b_{41}^{\prime \prime \prime}$ & -0.0095432 & 0.2427321 & 0.0079244 & -0.1546944 \\
\hline$b_{51}^{\prime \prime 1}$ & -0.2508516 & -0.1503851 & 0.1311359 & 0.0449006 \\
\hline \multirow[t]{2}{*}{$b_{61}^{\prime \prime \prime}$} & -0.0501051 & 0.1889811 & -0.0278058 & -0.0735363 \\
\hline & $A_{02}^{\prime}$ & $A_{12}^{\prime}$ & $A_{22}^{\prime}$ & $A_{32}^{\prime}$ \\
\hline \multicolumn{5}{|c|}{$m=2(K p: 3.0-4.5) k_{2}=3.75^{02}$} \\
\hline$b_{02}^{\prime}$ & 9.5988037 & 21.479304 & 3.1926672 & 1.9755171 \\
\hline$b_{12}^{\prime 2}$ & 5.1430543 & 6.6075450 & 1.2270596 & -0.3518031 \\
\hline$b_{22}^{\prime 12}$ & -1.7371661 & -1.2566572 & -0.0054540 & -0.0143734 \\
\hline$b_{32}^{\prime 2}$ & -0.2230598 & 0.3451542 & 0.1229414 & -0.0291659 \\
\hline
\end{tabular}


Appendix B (Continued)

\begin{tabular}{|c|c|c|c|c|}
\hline Units & {$\left[\operatorname{ergs~} \mathrm{cm}^{2} \cdot \mathrm{s}^{-1}\right]$} & {$\left[{ }^{0}\right]$} & {$\left[{ }^{0}\right]$} & {$\left[{ }^{\circ}\right]$} \\
\hline$b_{42}^{\prime}$ & 0.0422935 & -0.0564700 & 0.0573293 & -0.0337693 \\
\hline$b_{52}^{\prime \prime 2}$ & -0.0122696 & -0.1576626 & -0.0562889 & 0.0388444 \\
\hline$b_{62}^{\prime 2}$ & 0.0080510 & 0.2253359 & 0.0956040 & 0.0575712 \\
\hline$b_{12}^{\prime \prime}$ & -0.6726416 & 1.2164832 & 0.4361579 & -0.1477385 \\
\hline$b_{22}^{\prime \prime \prime}$ & -0.0727642 & -0.2300587 & -0.5028849 & -0.2390160 \\
\hline$b_{32}^{\pi \prime}$ & 0.1200389 & -0.3520732 & -0.2906078 & 0.1048181 \\
\hline$b_{42}^{\prime \prime \prime}$ & 0.0465696 & 0.2226716 & 0.0514080 & -0.1010366 \\
\hline$b_{52}^{\prime \prime \prime}$ & -0.4557674 & -0.0385575 & 0.0760109 & -0.0255308 \\
\hline$b_{62}^{\prime \prime \prime}$ & -0.1550104 & 0.1310600 & 0.0308203 & -0.0628866 \\
\hline & $A_{03}^{\prime}$ & $A_{13}^{\prime}$ & $A_{23}^{\prime}$ & $A_{33}^{\prime}$ \\
\hline \multicolumn{5}{|c|}{$m=3(K p: 4.5-6.0) k_{3}=5.25$} \\
\hline$b_{03}^{\prime}$ & 12.246801 & 23.511121 & 3.7091436 & 2.1764069 \\
\hline$b_{13}^{\prime \prime}$ & 7.0481969 & 6.1681312 & 1.3842879 & -0.4713056 \\
\hline$b_{23}^{\prime}$ & -3.0928509 & -0.7124293 & 0.1899543 & -0.0948302 \\
\hline$b_{33}^{\prime 3}$ & -1.2935699 & 0.2640897 & 0.0647574 & -0.1372124 \\
\hline$b_{43}^{\prime}$ & 0.0884275 & -0.2602568 & -0.0045988 & -0.0509513 \\
\hline$b_{53}^{\prime}$ & -0.0274394 & 0.0211500 & 0.0295777 & 0.0340843 \\
\hline$b_{63}^{\prime}$ & -0.2723690 & 0.2260950 & 0.1237925 & -0.0056936 \\
\hline$b_{13}^{\prime \prime}$ & -1.0729358 & 1.0434480 & 0.4866616 & -0.2832799 \\
\hline$b_{2}^{\prime \prime}$ & 0.1829594 & -0.3088645 & -0.4396647 & -0.0853116 \\
\hline$b_{33}^{\pi}$ & 0.6604424 & -0.6493409 & -0.3368631 & 0.0876408 \\
\hline$b_{43}^{\prime \prime \prime}$ & 0.1005881 & 0.3886247 & 0.2038886 & 0.0708100 \\
\hline$b_{53}^{\prime \prime \prime}$ & -0.3857214 & -0.0406112 & 0.0022551 & -0.0345108 \\
\hline$b_{63}^{\prime \prime \prime}$ & -0.3237388 & -0.0072146 & 0.0360084 & -0.0328183 \\
\hline & $A_{04}^{\prime}$ & $A_{14}^{\prime}$ & $A_{24}^{\prime}$ & $A_{34}^{\prime}$ \\
\hline \multicolumn{5}{|c|}{$m=4(K p: 6.0-8.0) k_{4}=7.0$} \\
\hline$b_{04}^{\prime}$ & 17.185531 & 27.197799 & 5.1669995 & 2.5417068 \\
\hline$b_{14}^{\prime}$ & 11.323595 & 5.3379375 & 1.1495666 & -0.6063337 \\
\hline$b_{24}^{\prime}$ & -1.2885997 & -1.1071272 & 0.0676676 & 0.0136708 \\
\hline$b_{34}^{\prime 2}$ & -0.8853908 & 0.3888829 & -0.1339100 & -0.0624044 \\
\hline$b_{44}^{\prime}$ & 1.2169522 & -0.4494379 & -0.0978956 & 0.0024086 \\
\hline$b_{54}^{\prime+}$ & 0.9936972 & -0.2346430 & -0.2247035 & 0.0623906 \\
\hline$b_{64}^{\prime}$ & 0.3355213 & 0.3541175 & 0.0702655 & 0.0125311 \\
\hline$b_{14}^{\prime \prime}$ & -1.8969096 & -0.7900455 & 0.0107553 & -0.3075880 \\
\hline$b_{24}^{\prime \prime 4}$ & -1.5002061 & 0.5940236 & 0.2318213 & 0.0774584 \\
\hline$b_{34}^{24}$ & -0.4722835 & -0.7037725 & -0.1692764 & -0.1040405 \\
\hline$b_{44}^{\prime \prime 4}$ & -1.8746630 & 0.4504852 & 0.5258857 & -0.0402230 \\
\hline$b_{54}^{\prime \prime 4}$ & -0.8323257 & 0.2860252 & 0.1850265 & -0.0989170 \\
\hline$b_{64}^{\prime \prime 4}$ & 0.1062067 & -0.4846608 & -0.2689677 & 0.0636382 \\
\hline & $A_{05}^{\prime}$ & $A_{15}^{\prime}$ & $A_{25}^{\prime}$ & $A_{35}^{\prime}$ \\
\hline \multicolumn{5}{|c|}{$m=5(K p: 8.0-10.) k_{5}=9.0$} \\
\hline$b_{05}^{\prime}$ & 33.435440 & 30.688625 & 5.9308809 & 4.1004123 \\
\hline$b_{15}^{\prime \prime}$ & 24.269584 & 3.9275374 & 0.3238901 & -0.4237273 \\
\hline$b_{25}^{\prime}$ & -4.0539849 & -2.4440639 & -0.9602533 & -0.1556447 \\
\hline$b_{35}^{\prime}$ & -5.0305214 & 1.8123949 & 0.2338884 & 0.2188714 \\
\hline$b_{45}^{\prime}$ & -1.7500538 & -0.2253542 & 0.4168486 & -0.0093918 \\
\hline$b_{55}^{\prime \prime}$ & -0.8196342 & 0.4044060 & -0.1198387 & -0.0095218 \\
\hline$b_{65}^{\prime \prime s}$ & -0.7520601 & -1.2894243 & -0.5288058 & 0.0896570 \\
\hline$b_{15}^{\prime \prime}$ & -5.9059449 & -2.6264976 & 0.8511741 & -0.6852183 \\
\hline$b_{25}^{\prime \prime}$ & -0.1670781 & 3.0068989 & 0.9358651 & -0.0488459 \\
\hline$b_{35}^{\prime \prime \prime}$ & 2.8623939 & -2.5030701 & -1.2186771 & 0.3298003 \\
\hline$b_{45}^{\prime \prime \prime}$ & 0.9153974 & 0.7056867 & 0.1745191 & 0.1314771 \\
\hline$b_{55}^{\prime \prime \prime}$ & 1.5992821 & -0.1550183 & -0.3299605 & -0.1826762 \\
\hline$b_{65}^{\prime \prime \prime}$ & 0.9162504 & -0.7857376 & -0.2456667 & 0.2985601 \\
\hline
\end{tabular}

